\title{
Alterstice
}

Revue internationale de la recherche interculturelle

International Journal of Intercultural Research

Revista International de la Investigacion Intercultural

\section{Construction de la littératie en santé des familles francophones en milieu linguistique minoritaire [synthèse en français]}

\section{Margareth Santos Zanchetta, Margot Kaszap, Mohamed Mohamed, Louise Racine, Christine Maheu, Diana Masny, Ines Cèsar, Claire Maltais, Ghislain Sangwa-Lugoma, Nancy Lussier et Diana Kinslikh}

Volume 2, numéro 2, 2012

Regards pluriels sur les interventions sociales et de santé en contexte de diversité

URI : https://id.erudit.org/iderudit/1077565ar

DOI : https://doi.org/10.7202/1077565ar

Aller au sommaire du numéro

Éditeur(s)

Alterstice

ISSN

1923-919X (numérique)

Découvrir la revue

Citer ce document

Zanchetta, M. S., Kaszap, M., Mohamed, M., Racine, L., Maheu, C., Masny, D., Cèsar, I., Maltais, C., Sangwa-Lugoma, G., Lussier, N. \& Kinslikh, D. (2012). Construction de la littératie en santé des familles francophones en milieu linguistique minoritaire [synthèse en français]. Alterstice, 2(2), i-iv. https://doi.org/10.7202/1077565ar

(C) Margareth Santos Zanchetta, Margot Kaszap, Mohamed Mohamed, Louise Racine, Christine Maheu, Diana Masny, Ines Cèsar, Claire Maltais, Ghislain Sangwa-Lugoma, Nancy Lussier et Diana Kinslikh, 2012
Ce document est protégé par la loi sur le droit d'auteur. L'utilisation des services d'Érudit (y compris la reproduction) est assujettie à sa politique d'utilisation que vous pouvez consulter en ligne.

https://apropos.erudit.org/fr/usagers/politique-dutilisation/ 


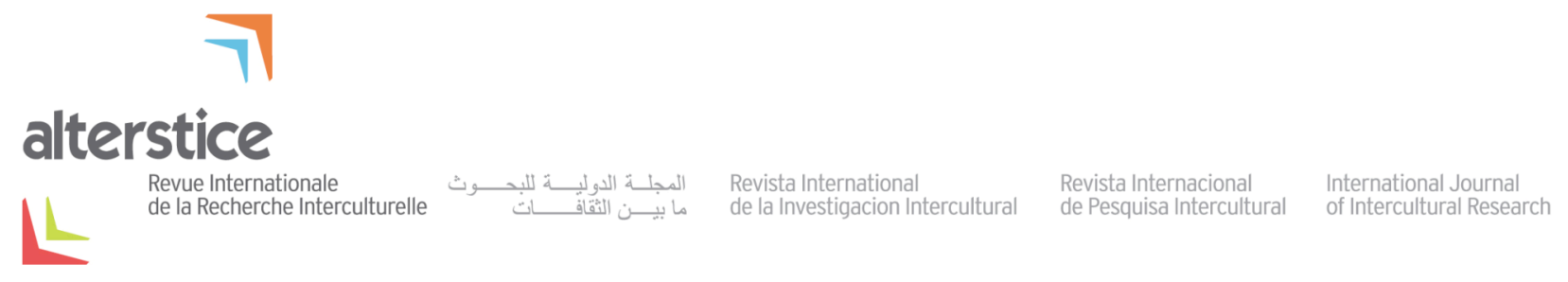

ARTICLE THÉMATIQUE

\title{
Construction de la littératie en santé des familles francophones en milieu linguistique minoritaire [synthèse en français*]
}

Margareth Santos Zanchetta ${ }^{1}$, Margot Kaszap ${ }^{2}$, Mohamed Mohamed ${ }^{3}$, Louise Racine ${ }^{4}$, Christine Maheu ${ }^{5}$, Diana Masny $^{6}$, Ines Cèsar ${ }^{7}$, Claire Maltais ${ }^{6}$, Ghislain Sangwa-Lugoma $^{8}$, Nancy Lussier ${ }^{1}$, Diana Kinslikh ${ }^{9}$

\author{
Rattachement des auteurs \\ ${ }^{1}$ Ryerson University, Toronto, Canada; ${ }^{2}$ Université Laval, Québec, Canada; ${ }^{3}$ Toronto Public Health, Toronto, Canada; \\ ${ }^{4}$ University of Saskatchewan, Saskatoon, Canada; ${ }^{5}$ McGill University, Montréal, Canada; ${ }^{6}$ University of Ottawa, \\ Ottawa, Canada; ${ }^{7}$ ABC-Head Start Society, Edmonton, Canada; ${ }^{8}$ University of Alberta, Edmonton, Canada; ${ }^{9}$ West \\ Park Healthcare Centre, Toronto, Canada.
}

\section{Correspondance}

mzanchet@ryerson.ca

\section{Mots clés}

minorité linguistique; immigrant; francophone; littératie familiale en santé; vulnérabilité linguistique

\section{Pour citer cet article}

Zanchetta, M., Kaszap, M., Mohamed, M., Racine, L., Maheu, C., Masny, D., Cèsar, I., Maltais, C., Sangwa-Lugoma, G., Lussiers, N. et Kinslikh, D. (2012). Construction de la littératie en santé des familles francophones en milieu linguistique minoritaire [synthèse en français]. Alterstice, 2(2), i-iv. [Construction of Francophone families' health literacy in a linguistic minority situation]

*L'article complet en anglais est disponible sur le site d'Alterstice.

\section{Introduction}

Une mobilité accrue des populations exige que les barrières de langues ne soient plus ignorées par le système de santé. Cependant, le lien qui existe entre les barrières linguistiques et la littératie en santé a déjà été démontré mais n'est pas encore reconnu (Bâ, Rivard et Levesque, 2005; Bouchard, Gilbert, Landry et Deveau, 2006). La littératie en santé est constituée d'actifs venant de la famille, de l'école, du milieu social, de la culture et des expériences professionnelles; ils s'accumulent au fil des ans et se traduisent en connaissances, croyances, attitudes, peurs et comportements de santé (Kaszap et Zanchetta, 2009).

\section{Buts de l'étude}

Cette étude visait à décrire la littératie familiale en santé dans un nouveau contexte linguistique, celui des immigrants et des francophones nés au Canada vivant en contexte de minorité linguistique. 


\section{Questions de recherche}

1) Quelle dynamique y a-t-il entre les expériences sociales préliminaires et les pratiques courantes de santé, développés par les familles pour assurer leur bien-être tout en apprenant sur la santé?

2) Quelles stratégies les immigrants et francophones nés au Canada utilisent-ils pour augmenter leurs connaissances en santé afin de pourvoir au bien-être de leur famille?

3) Comment les pratiques familiales de santé changent-elles afin de faire face aux contraintes structurales et linguistiques?

\section{Cadre théorique}

La théorie des littératies multiples (Masny, 2010) forme le cadre théorique principal de cette étude, incorporant la pédagogie de la libération et celle de la conscience critique (Freire, 1973). Ces théories permettent de relier le concept d'identité linguistique francophone avec celui de littératie en santé, à travers les connaissances intergénérationnelles transmises et l'incorporation de valeurs, de croyances et de pratiques de la vie courante en santé modulées par toutes formes de langue parlée et toute lecture portant un regard sur le monde.

\section{Méthode}

Cette étude qualitative a été menée avec un échantillon de convenance de 28 participants, qui ont relaté leur histoire familiale, cette dernière touchant 95 francophones. Les critères d'inclusion étaient

- avoir le français comme langue parlée à la maison,

- être d'origine multiculturelle ou

- être né au Canada mais vivre dans une situation de minorité linguistique hors Québec,

- recevoir des services de santé ou sociaux.

Des entrevues semi-structurées ont permis de recueillir une auto-évaluation de la littératie en santé des participants et d'explorer les énoncés de leur expérience systémique en santé (Wright et Leahey, 2005). Le processus analytique (Paillé et Mucchielli, 2008) a généré des thématiques permettant de produire un haut niveau d'abstraction et de définir cinq catégories conceptuelles. Ces catégories ont été réduites à leur signification épistémologique propre afin de répondre à la question : comment la construction de la littératie familiale en santé se construit-elle dans des situations de minorité linguistique?

\section{Résultats}

Les résultats présentés relatent les impacts majeurs relevés dans les entrevues familiales concernant les expériences de santé, les pratiques des six derniers mois précédant l'entrevue ainsi que l'expérience de littératie en santé. Les résultats décrivent comment les expériences en santé des familles d'immigrants francophones ont influencé leurs pratiques de santé courantes, leurs stratégies pour améliorer leurs connaissances en santé et leurs façons de gérer les contraintes socio-environnementales. Les répondants ont également présenté des suggestions pour améliorer l'accès des francophones au système de santé au Canada.

L'analyse a relevé cinq thèmes ayant permis de clarifier la littératie familiale en santé des participants vivant en situation de minorité linguistique :

- I'utilisation de pratiques traditionnelles pour maintenir sa santé,

- l'exploration de l'environnement social,

- l'interaction formelle et informelle avec les réseaux sociaux,

- la consternation face à l'iniquité d'accès en matière de santé,

- l'acceptation ou l'indignation face au manque de service de santé en français. 
Seuls les participants bilingues et habiles avec l'utilisation de l'ordinateur étaient conscients du manque d'information en santé en français dans le réseau Internet. L'organisation du système de santé pouvant soutenir et desservir la population grandissante d'immigrants francophones dépend des fonds gouvernementaux provinciaux. Nos résultats confirment que l'isolement linguistique des francophones n'est pas simplement géographique. Nous avons même trouvé un faible niveau de littératie en santé chez des immigrants francophones bilingues, qui se sont sentis dépourvus lorsqu'ils ont eu à communiquer en anglais dans des moments d'émotion intense ayant affecté leur santé ou celle de leurs proches.

La vulnérabilité linguistique des participants s'est accrue au fil du temps selon quatre facteurs :

- un accès minimal aux services de santé et d'information en français (celle-ci provient seulement de leur médecin de famille);

- un manque de motivation pour améliorer leur santé, à moins d'une maladie soudaine, et par voie de conséquence un manque de connaissances en santé;

- dans la recherche d'information sur la santé, un manque de critique sur la qualité de l'information, et par conséquent une augmentation du risque de prendre une mauvaise décision

- l'atténuation possible de la vulnérabilité par l'apprentissage de l’anglais.

\section{Discussion}

Nos résultats apportent une compréhension alternative à l'impression véhiculée selon laquelle l'immigrant est une personne en parfaite santé. Les francophones vivant dans des situations de minorité linguistique sont plutôt probablement invisibles dans les données du réseau de la santé. II se peut que les immigrants francophones sousestiment la sévérité de leurs symptômes et ne cherchent pas à consulter, soit par manque de connaissances pour s'orienter et recevoir des soins, soit par manque de services en français. Le fait de ne pas être au courant de l'existence des services de santé communautaires a comme conséquence que les immigrants ont plutôt tendance à n'accéder au système de santé que par les médecins de famille ou les départements d'urgences. Les immigrants qui ne se heurtent pas à la barrière de la langue ont plutôt tendance à utiliser les services communautaires comme porte d'entrée du système de santé.

Les résultats illustrent aussi que l'oppression historique, culturelle et politique vécue par les immigrants francophones au Canada génère un processus d'exclusion : ils se sentent comme étant "les autres ". La barrière de la langue des participants, une fois documentée, révèle que le mythe de "l'immigrant en santé " n’est pas réel mais correspond plutôt à une invisibilité dans le système de santé canadien dû à un piètre accès aux services de santé.

\section{Conclusion}

L'isolement linguistique et le manque de connaissances des organisations locales culturelles chez les immigrants francophones sont deux faits saillants de l'étude. Ces deux points illustrent l'importance pour les promoteurs de la santé et des services sociaux de combiner leurs efforts afin d'aider les nouveaux arrivant à surmonter les défis de l'accès au système de santé.

\section{Références bilbiographiques}

Bâ, O., Rivard, L. et Levesque, A. (2005). Les immigrants francophones en milieu minoritaire - services, défis, obstacles. Winnipeg : Collège Universitaire de Saint-Boniface.

Bouchard, L., Gilbert, A., Landry, R. et Deveau, K. (2006). Capital social, santé et minorités francophones. Revue Canadienne de Santé Publique, 97, S17-S21.

Freire, P. (1973). Education for critical consciousness. New York : Continuum. 
Kaszap, M. et Zanchetta, M. S. (2009). La littératie en santé, vécue dans la simplicité mais comprise à travers la complexité : regard sur les communautés culturelles (francophones, minoritaires et multiethniques). Dans Masny, D. (dir.), Les littératies multiples : lire au $21^{e}$ siècle (pp. 287-325). Ottawa : Les Presses de l'Université d'Ottawa.

Masny, D. (2010). Multiple literacies theory: How it functions, what it produces. Perspectiva, 28, $337-352$.

Paillé, P. et Mucchielli, A. (2008). L'analyse qualitative en sciences humaines et sociales ( $2^{\mathrm{e}}$ éd.). Paris : Armand Colin.

Wright, L. et Leahey, M. (2005). Nurses and Families: A guide for family assessment and intervention ( $4^{\mathrm{e}}$ éd.). Philadelphia, PA : F. A. Davis. 